DO-TH 95/20, THES-TP 95/14

(hep-ph/9512333)

\title{
Goldstone modes and the Higgs condensation beyond one loop
}

\author{
G. Cvetič \\ Inst. für Physik, Universität Dortmund, 44221 Dortmund, Germany \\ N.D. Vlachos \\ Dept. of Theor. Physics, Aristotle University of Thessaloniki, 54006 Thessaloniki, Greece
}

\begin{abstract}
We study the Higgs condensation $H=\langle\bar{t} t\rangle$ mechanism in the Top-mode Standard Model at the next-to-leading order in $1 / N_{\mathrm{c}}$. The calculation includes the effects of the Goldstone fields, but not the effects of the transverse components of the electroweak gauge bosons. The resulting effective theory is parametrized by means of a finite energy cut-off $\Lambda$ at which the condensation is supposed to take place. Demanding that the next-to-leading order contributions not dominate over the leading order ones, we get a rather low bound for the cut-off: $\Lambda=\mathcal{O}(1 \mathrm{TeV})$. QCD effects can change the results somewhat, but the basic conclusions remain unchanged. The inclusion of the Goldstone degrees of freedom tends to decrease the bound on $\Lambda$.
\end{abstract}

PACS number(s): 12.60.Rc, 14.80.Bn, 11.15.Pg

\section{Introduction}

The idea that the Higgs mesons could be bound states of heavy quark pairs has been developed and worked on in a series of papers by various authors ( [1]- [4, and references therein), motivated by an earlier work of Nambu and Jona-Lasinio (NJL) [5]. The bound states (condensates) are treated in these works either in the leading- $N_{\mathrm{c}}$ approximation, or in a form that takes into account part of the effects beyond the leading- $N_{\mathrm{c}}$ - by using improved Schwinger-Dyson equations, or renormalization 
group equations (RGEs). A particularly transparent NJL-type framework, containing the essential features of the mentioned idea of condensation, is the Top-mode Standard Model (TSM) Lagrangian, known also as the BHL (Bardeen-Hill-Lindner) Lagrangian [3].

In a recent work [6], we studied the next-to-leading order (ntl) contributions in the $\left(1 / N_{\mathrm{c}}\right)$ expansion in the TSM by including quadratic fluctuations of the composite Higgs $H=\langle\bar{t} t\rangle$ in the effective potential $V_{\text {eff }}$. The existence of a non-trivial minimum in the effective potential led us to the conclusion that the cut-off $\Lambda$ is bound from above: $\Lambda \leq \Lambda_{\text {crit }} \approx 4.7 m_{t}^{\text {phys }}$ for $N_{\mathrm{c}}=3$. In [6], contributions related to components of the massive electroweak gauge bosons were not considered. QCD effects were included, but their impact was found to be small. We considered the effective potential as a function of a hard mass term $\lambda \sigma_{0}$ of the top quark, parametrized by the expectation value $\sigma_{0}$ of a composite (initially auxiliary) scalar field $\sigma$.

In the present work, we continue the work of ref. [6]. We show that the inclusion of the "scalar" longitudinal degrees of freedom of the electroweak gauge bosons $W$ and $Z$ (i.e., of the three composite Goldstones) at the ntl-level does change the numerics substantially, but does not change the basic conclusion of the paper [6]. The cut-off remains in the region $\mathcal{O}(1 \mathrm{TeV})$. As a matter of fact, the Goldstone contributions at the ntl-level tend to decrease the cut-off even further.

\section{The model and the effective potential}

In the Top-mode Standard Model (TSM) Lagrangian [3], a truncated gauge-invariant 4-fermion interaction at a high energy scale $E \sim \Lambda$ is assumed to be responsible for the creation of a composite Higgs field $H=\langle\bar{t} t\rangle$

$$
\mathcal{L}=\mathcal{L}_{\text {kin }}^{0}+G\left(\bar{\Psi}_{\mathrm{L}}^{i a} t_{\mathrm{R} a}\right)\left(\bar{t}_{\mathrm{R}}^{b} \Psi_{\mathrm{L} b}^{i}\right) \quad \text { for } E \sim \Lambda
$$

Here, $a$ and $b$ are the color and $i$ the isospin indices, $\Psi_{\mathrm{L}}^{T}=\left(t_{\mathrm{L}}, b_{\mathrm{L}}\right)$, and $\mathcal{L}_{\text {kin }}^{0}$ contains the usual gauge-invariant kinetic terms for fermions and gauge bosons. The Lagrangian (11) leads to an effective framework for the minimal Standard Model. It can be rewritten in terms of an additional, as yet auxiliary, scalar isodoublet $\Phi$, by adding to it the following quadratic term $\square$

$$
\begin{gathered}
\mathcal{L}_{\text {new }}=\mathcal{L}_{\text {old }}-\left[M_{0} \tilde{\Phi}^{i \dagger}+\sqrt{G} \bar{\Psi}_{\mathrm{L}}^{i a} t_{\mathrm{R} a}\right]\left[M_{0} \tilde{\Phi}^{i}+\sqrt{G} \bar{t}_{\mathrm{R}}^{b} \Psi_{\mathrm{L} b}^{i}\right] \\
\text { where: } \tilde{\Phi}=i \tau_{2} \Phi^{*}, \quad \Phi=\frac{1}{\sqrt{2}}\left(\begin{array}{c}
\sqrt{2} \mathcal{G}^{+} \\
\mathcal{H}+i \mathcal{G}^{(0)}
\end{array}\right), \quad \mathcal{G}^{ \pm}=\frac{1}{\sqrt{2}}\left(\mathcal{G}^{(1)} \pm i \mathcal{G}^{(2)}\right) .
\end{gathered}
$$

\footnotetext{
${ }^{1}$ Addition of such a term changes the generating functional only by an irrelevant source-independent factor $[7]$.
} 
The resulting Lagrangian reads

$$
\begin{aligned}
\mathcal{L}= & i \bar{\Psi}^{a} \not \partial \Psi_{a}-\frac{M_{0} \sqrt{G}}{\sqrt{2}}\left[\mathcal{H} \bar{t}^{a} t_{a}-i \mathcal{G}^{(0)} \bar{t}^{a} \gamma_{5} t_{a}\right]+\frac{M_{0} \sqrt{G}}{2}\left[\mathcal{G}^{+} \bar{t}^{a}\left(1-\gamma_{5}\right) b_{a}+\mathcal{G}^{-} \bar{b}^{a}\left(1+\gamma_{5}\right) t_{a}\right] \\
& -\frac{1}{2} M_{0}^{2}\left(\mathcal{H}^{2}+\mathcal{G}^{(0) 2}+2 \mathcal{G}^{-} \mathcal{G}^{+}\right)
\end{aligned}
$$

where $\mathcal{H}, \mathcal{G}^{(0)}, \mathcal{G}^{(1)}$ and $\mathcal{G}^{(2)}$ are the Higgs and the three real Goldstone components of the auxiliary complex isodoublet field $\Phi$, and $M_{0}$ is an unspecified bare mass term for $\Phi$ (at $E \sim \Lambda$ ) 2. These fields will eventually become the physical Higgs and the "scalar" longitudinal components of the massive electroweak bosons through quantum effects. We ignore in (3) the transverse components of $W^{ \pm}$and $Z$ and all the lighter quarks which we assume to be and remain massless. It can be shown that the massless Goldstones discussed here correspond to the Goldstone degrees of freedom of $W^{ \pm}$and $Z$ in the Landau gauge $(\xi \rightarrow \infty)$; incidentally, in this gauge, the ghosts do not couple to the scalar degrees of freedom and therefore they (the ghosts) do not contribute to the effective potential [8].

The effective potential $V_{\text {eff }}\left(H_{0}\right)$ of the Higgs field $\mathcal{H}$ can then be calculated in Euclidean space by means of the following formula

$$
\begin{aligned}
& \exp [\left.-\Omega V_{\mathrm{eff}}\left(H_{0}\right)\right]=\mathrm{const} \times \int \prod_{j=0}^{2}\left[\mathcal{D} \mathcal{G}^{(j)} \delta\left(\int d^{4} \bar{y} \mathcal{G}^{(j)}(\bar{y})\right)\right] \times \\
& \times \int \mathcal{D} \mathcal{H} \delta\left(\int d^{4} \bar{y} \mathcal{H}(\bar{y})-\Omega H_{0}\right) \int \mathcal{D} \bar{\Psi} \mathcal{D} \Psi \exp \left[+\int \mathcal{L} d^{4} \bar{x}\right],
\end{aligned}
$$

where we set $\hbar=1$. The bars over space-time components, derivatives and momenta from now on denote Euclidean quantities. $\Omega$ is the 4-dimensional volume (formally infinite). We note that the effective potential is the energy density of the ground state when the order parameters $H_{0}=\langle\mathcal{H}\rangle$ and $\left\langle\mathcal{G}^{(j)}\right\rangle=0(j=0,1,2)$ are kept fixed. Next, we integrate out the quark degrees of freedom, and expand the resulting expression in powers of $h(\bar{x})=\mathcal{H}(\bar{x})-H_{0}, \mathcal{G}^{(j)}(\bar{x})(j=0,1,2)$, including up to quadratic fluctuations. We thus obtain

$$
\begin{aligned}
& \Omega V_{\text {eff }}\left(H_{0}\right)=\frac{1}{2} \Omega M_{0}^{2} H_{0}^{2}-\operatorname{Tr} \ln \hat{B}_{0}-\ln \int_{-\infty}^{\infty} \prod_{j=0}^{2} d J_{j} \int_{-\infty}^{\infty} d J_{h} \int \prod_{j=0}^{2} \mathcal{D} \mathcal{G}^{(j)} \int \mathcal{D} h \\
& \quad \exp \left\{-\frac{M_{0}^{2}}{2} \int d^{4} \bar{x}\left[h^{2}+\mathcal{G}^{(0) 2}+2 \mathcal{G}^{+} \mathcal{G}^{-}\right]-\frac{1}{2} \operatorname{Tr}\left(\hat{B}_{0}^{-1} \delta \hat{B}\right)^{2}-i \sum_{j=0}^{2} J_{j} \int d^{4} \bar{x} \mathcal{G}^{(j)}-i J_{h} \int d^{4} \bar{x} h\right\}(5)
\end{aligned}
$$

where the integrals over $J_{j}(j=0,1,2)$ and $J_{h}$ represent the corresponding $\delta$-functions in (đ), and the translationally invariant operator $\hat{B}_{0}$ as well as the scalar fluctuation operator $\delta \hat{B}$ can be written in

\footnotetext{
2 The physical results will be independent of the value of $M_{0}^{2}$.
} 
the $\bar{x}$-basis as

$$
\begin{aligned}
\left\langle\bar{x}^{\prime} ; a\left|\hat{B}_{0}\right| \bar{x} ; b\right\rangle & =\delta_{a b}\left[\begin{array}{cc}
\left(i \bar{\phi}+g_{0} H_{0} / \sqrt{2}\right) & 0 \\
0 & i \bar{\phi}
\end{array}\right] \delta\left(\bar{x}-\bar{x}^{\prime}\right), \\
\left\langle\bar{x}^{\prime} ; a|\delta \hat{B}| \bar{x} ; b\right\rangle & =\delta_{a b} \frac{g_{0}}{\sqrt{2}}\left[\begin{array}{cc}
\left(h-i \gamma_{5} \mathcal{G}^{(0)}\right) & -\mathcal{G}^{+}\left(1-\gamma_{5}\right) / \sqrt{2} \\
-\mathcal{G}^{-}\left(1+\gamma_{5}\right) / \sqrt{2} & 0
\end{array}\right] \delta\left(\bar{x}-\bar{x}^{\prime}\right) .
\end{aligned}
$$

In (6), $a$ and $b$ are color indices, $g_{0}=M_{0} \sqrt{G}$, and the $2 \times 2$ matrices are in isospin space. The first two terms on the r.h.s. of (5) represent the leading- $N_{\mathrm{c}}$ contribution $V_{\mathrm{eff}}^{(0)}$ to the effective potential, while the exponential terms related to the quadratic fluctuations of the scalar fields lead to the full next-to-leading (ntl) contribution $V_{\mathrm{eff}}^{(1)}$. The path integrals corresponding to these terms are of the Gaussian type and can be explicitly evaluated. Proceeding in close analogy to [6] [3, we obtain the following:

$$
V_{\mathrm{eff}}=V_{\mathrm{eff}}^{(0)}+V_{\mathrm{eff}}^{(1)}+\mathcal{O}\left(1 / N_{\mathrm{c}}^{2}\right)
$$

where the leading- $N_{\mathrm{c}}$ contribution is

$$
V_{\mathrm{eff}}^{(0)}\left(\lambda^{2} \sigma_{0}^{2}\right)=\sigma_{0}^{2}-\frac{N_{\mathrm{c}}}{8 \pi^{2}} \int_{0}^{\Lambda_{f}^{2}} d \bar{k}^{2} \bar{k}^{2} \ln \left[1+\frac{\lambda^{2} \sigma_{0}^{2}}{\bar{k}^{2}}\right],
$$

and the ntl-term is

$$
V_{\mathrm{eff}}^{(1)}\left(\lambda^{2} \sigma_{0}^{2}\right)=\left[\frac{1}{2 \Omega} \operatorname{Tr} \ln \left(\hat{A}_{\mathrm{n}}\right)_{11}+\frac{1}{2 \Omega} \operatorname{Tr} \ln \left(\hat{A}_{\mathrm{n}}\right)_{22}+\frac{2}{2 \Omega} \operatorname{Tr} \ln \left(\hat{A}_{\mathrm{ch}}\right)\right] .
$$

We denoted

$$
\lambda=\sqrt{G}, \quad \sigma_{0}=M_{0} H_{0} / \sqrt{2},
$$

and $\hat{A}_{\mathrm{n}}$ and $\hat{A}_{\mathrm{ch}}$ are the kernels of the Gaussian path integrals corresponding to the contributions of the neutral scalars $\mathcal{H}, \mathcal{G}^{(0)}$, and charged scalars $\mathcal{G}^{ \pm}$, respectively:

$$
\left\langle\bar{x}^{\prime}\left|\hat{A}_{\mathrm{n}, \mathrm{ch}}\right| \bar{x}\right\rangle=\frac{1}{(2 \pi)^{4}} \int d^{4} \bar{p} \exp \left[i \bar{p}\left(\bar{x}-\bar{x}^{\prime}\right)\right] \tilde{A}_{\mathrm{n}, \mathrm{ch}}\left(\bar{p}^{2}\right) .
$$

The Fourier transforms of these kernels are

$$
\begin{aligned}
& \tilde{A}_{\mathrm{n}, \mathrm{ch}}\left(\bar{p}^{2}\right)=\int d^{4} \bar{x} \exp [-i \bar{p} \cdot \bar{x}]\left\langle 0\left|\hat{A}_{\mathrm{n}, \mathrm{ch}}\right| \bar{x}\right\rangle=2\left[\hat{1}-2 \lambda^{2} N_{\mathrm{c}} \mathcal{K}_{\mathrm{n}, \mathrm{ch}}\left(\bar{p}^{2} ; \lambda^{2} \sigma_{0}^{2}\right)\right], \\
& \mathcal{K}_{\mathrm{n}}=\frac{1}{4} \int_{\bar{k}^{2} \leq \Lambda_{\mathrm{f}}^{2}} \frac{d^{4} \bar{k}}{(2 \pi)^{4}}\left[\begin{array}{cc}
\operatorname{tr}_{\mathrm{f}}\left(\frac{i}{\left(\bar{k}-\lambda \sigma_{0}\right)} \frac{i}{\left(\not{p}+\not{k}-\lambda \sigma_{0}\right)}\right) & 0 \\
0 & \operatorname{tr}_{\mathrm{f}}\left(\frac{i}{\left(\vec{k}-\lambda \sigma_{0}\right)}\left(-i \gamma_{5}\right) \frac{i}{\left(\not{p}+\bar{k}-\lambda \sigma_{0}\right)}\left(-i \gamma_{5}\right)\right)
\end{array}\right], \\
& \mathcal{K}_{\mathrm{ch}}=\frac{1}{2} \int_{\bar{k}^{2} \leq \Lambda_{\mathrm{f}}^{2}} \frac{d^{4} \bar{k}}{(2 \pi)^{4}} \operatorname{tr}_{\mathrm{f}}\left[\frac{i}{\left(\overline{\not h}-\lambda \sigma_{0}\right)}\left(\frac{1-\gamma_{5}}{2}\right) \frac{i}{(\not{\not p}+\overline{\not k})}\left(\frac{1+\gamma_{5}}{2}\right)\right] .
\end{aligned}
$$

\footnotetext{
${ }^{3}$ In [6] we used for the ntl-contribution the notation $V_{\mathrm{eff}}^{(\mathrm{ntl})}$ (here: $V_{\mathrm{eff}}^{(1)}$ ), and for the leading- $N_{\mathrm{c}}$ (i.e., $0+1$-loop) contribution the notation $V_{\mathrm{eff}}^{(0)}+V_{\mathrm{eff}}^{(1)}$ (here: $\left.V_{\mathrm{eff}}^{(0)}\right)$.
} 
Here, $\operatorname{tr}_{\mathrm{f}}$ means tracing over the $4 \times 4$ spinor matrices. The integrals in (12), as well as the one in (8), are regularized by means of a simple spherical cut-off $\Lambda_{\mathrm{f}}$ for the fermionic (top quark) momenta $|\bar{k}|$. We note that $\left(\mathcal{K}_{\mathrm{n}}\right)_{11}$ and $\left(\mathcal{K}_{\mathrm{n}}\right)_{22}$ are truncated 2-point Green functions corresponding to a $(t \bar{t})$-loop carrying two external Higgs legs, and two neutral Goldstone legs (with momentum $\bar{p}$ ), respectively. Analogously, $\mathcal{K}_{\mathrm{ch}}$ corresponds to a $(b \bar{t})$-loop with two external legs of the charged Goldstones. The tracing over the colors led to factors $N_{\mathrm{c}}(=3)$ in $V_{\text {eff }}^{(0)}$ and in front of $\mathcal{K}_{\mathrm{n}, \mathrm{ch}}$ in $V_{\mathrm{eff}}^{(1)}$. The tracing in $(9)$ is over spinor space and the momentum basis, involving a second integral over the bosonic momenta $\bar{p}$ (cf. also ref. [6]). We introduce for these momenta a second spherical cut-off: $\bar{p}^{2} \leq \Lambda_{\mathrm{b}}^{2}$, where subscript "b" stands for "bosonic" (note: $\Lambda_{\mathrm{b}} \sim \Lambda_{\mathrm{f}}$ ). We then rescale all the momenta $\left\{\bar{k}^{2}, \bar{p}^{2}\right\} \rightarrow \Lambda_{\mathrm{f}}^{2}\left\{\bar{k}^{2}, \bar{p}^{2}\right\}$, and introduce the following dimensionless quantities:

$$
\begin{gathered}
\varepsilon^{2}=\lambda^{2} \frac{\sigma_{0}^{2}}{\Lambda_{\mathrm{f}}^{2}}=\frac{G M_{0}^{2}}{2 \Lambda_{\mathrm{f}}^{2}} H_{0}^{2}, \quad a=\frac{\left(G N_{\mathrm{c}} \Lambda_{\mathrm{f}}^{2}\right)}{\left(8 \pi^{2}\right)}, \\
\Xi_{\mathrm{eff}}=8 \pi^{2} V_{\mathrm{eff}} /\left(N_{\mathrm{c}} \Lambda_{\mathrm{f}}^{4}\right)=\Xi^{(0)}+\frac{1}{N_{\mathrm{c}}} \Xi^{(1)}+\mathcal{O}\left(\frac{1}{N_{\mathrm{c}}^{2}}\right) .
\end{gathered}
$$

The resulting expressions for the leading- $N_{\mathrm{c}}$ term $\Xi^{(0)}$ and the ntl-term $\Xi^{(1)}$ are

$$
\begin{gathered}
\Xi^{(0)}\left(\varepsilon^{2} ; a\right)=\frac{\varepsilon^{2}}{a}-\int_{0}^{1} d \bar{k}^{2} \bar{k}^{2} \ln \left(1+\frac{\varepsilon^{2}}{\bar{k}^{2}}\right) \\
\Xi^{(1)}\left(\varepsilon^{2} ; \Lambda_{\mathrm{b}}^{2} / \Lambda_{\mathrm{f}}^{2} ; a\right)=\left\{\frac{1}{4} \int_{0}^{\Lambda_{\mathrm{b}}^{2} / \Lambda_{\mathrm{f}}^{2}} d \bar{p}^{2} \bar{p}^{2} \ln \left[1-a \mathcal{J}_{H}\left(\bar{p}^{2} ; \varepsilon^{2}\right)\right]\right. \\
\left.+\frac{1}{4} \int_{0}^{\Lambda_{\mathrm{b}}^{2} / \Lambda_{\mathrm{f}}^{2}} d \bar{p}^{2} \bar{p}^{2} \ln \left[1-a \mathcal{J}_{G n}\left(\bar{p}^{2} ; \varepsilon^{2}\right)\right]+\frac{2}{4} \int_{0}^{\Lambda_{\mathrm{b}}^{2} / \Lambda_{\mathrm{f}}^{2}} d \bar{p}^{2} \bar{p}^{2} \ln \left[1-a \mathcal{J}_{G c h}\left(\bar{p}^{2} ; \varepsilon^{2}\right)\right]\right\} .
\end{gathered}
$$

Subscripts $H, G n$ and $G c h$ correspond to contributions from the Higgs, neutral Goldstone and charged Goldstone degrees of freedom, respectively. The dimensionless 2-point Green functions are defined as

$$
\begin{aligned}
\mathcal{J}_{H}\left(\bar{p}^{2} ; \varepsilon^{2}\right) & =\frac{16 \pi^{2}}{\Lambda_{\mathrm{f}}^{2}} \mathcal{K}_{\mathrm{n}}\left(\Lambda_{\mathrm{f}}^{2} \bar{p}^{2} ; \lambda^{2} \sigma_{0}^{2}\right)_{11}=\frac{1}{\pi^{2}} \int_{\bar{k}^{2} \leq 1} d^{4} \bar{k} \frac{\left[\bar{k} \cdot(\bar{p}+\bar{k})-\varepsilon^{2}\right]}{\left(\bar{k}^{2}+\varepsilon^{2}\right)\left[(\bar{p}+\bar{k})^{2}+\varepsilon^{2}\right]}, \\
\mathcal{J}_{G n}\left(\bar{p}^{2} ; \varepsilon^{2}\right) & =\frac{16 \pi^{2}}{\Lambda_{\mathrm{f}}^{2}} \mathcal{K}_{\mathrm{n}}\left(\Lambda_{\mathrm{f}}^{2} \bar{p}^{2} ; \lambda^{2} \sigma_{0}^{2}\right)_{22}=\frac{1}{\pi^{2}} \int_{\bar{k}^{2} \leq 1} d^{4} \bar{k} \frac{\left[\bar{k} \cdot(\bar{p}+\bar{k})+\varepsilon^{2}\right]}{\left(\bar{k}^{2}+\varepsilon^{2}\right)\left[(\bar{p}+\bar{k})^{2}+\varepsilon^{2}\right]}, \\
\mathcal{J}_{G c h}\left(\bar{p}^{2} ; \varepsilon^{2}\right) & =\frac{16 \pi^{2}}{\Lambda_{\mathrm{f}}^{2}} \mathcal{K}_{\mathrm{ch}}\left(\Lambda_{\mathrm{f}}^{2} \bar{p}^{2} ; \lambda^{2} \sigma_{0}^{2}\right)_{11}=\frac{1}{\pi^{2}} \int_{\bar{k}^{2} \leq 1} d^{4} \bar{k} \frac{\bar{k} \cdot(\bar{p}+\bar{k})}{\left(\bar{k}^{2}+\varepsilon^{2}\right)(\bar{p}+\bar{k})^{2}} .
\end{aligned}
$$

The expressions for $\Xi^{(0)}$ and $\Xi^{(1)}$ can also be rederived diagrammatically by summing up terms corresponding to the 1-PI Green functions depicted in Figs. 1 and 2 (cf. also [6]).

The minimization of the leading- $N_{\mathrm{c}}$ part of $\Xi_{\text {eff }}$ leads to the familiar leading- $N_{\mathrm{c}}$ gap equation connecting the cut-off $\Lambda_{\mathrm{f}}$, the 4 -fermion coupling strength $G$ and the leading- $N_{\mathrm{c}}$ approximation $m_{t}^{(0)}$ 
to the mass of the top quark

$$
\begin{gathered}
\left.\frac{\partial \Xi^{(0)}\left(\varepsilon^{2} ; a\right)}{\partial \varepsilon^{2}}\right|_{\varepsilon^{2}=\varepsilon_{0}^{2}}=0, \Rightarrow \\
\Rightarrow \quad a \quad\left(=\frac{G N_{\mathrm{c}} \Lambda_{\mathrm{f}}^{2}}{8 \pi^{2}}\right)=\left[1-\varepsilon_{0}^{2} \ln \left(\varepsilon_{0}^{-2}+1\right)\right]^{-1}(=\mathcal{O}(1)), \quad \text { where: } \varepsilon_{0}^{2}=\left(m_{t}^{(0)} / \Lambda_{\mathrm{f}}\right)^{2} .
\end{gathered}
$$

This condition shows that the parameter $a(>1)$ is a number of $\mathcal{O}(1)$, and should be regarded as a quantity $\mathcal{O}\left(N_{\mathrm{c}}^{0}\right)$ in the $\left(1 / N_{\mathrm{c}}\right.$ )-expansion, as already done in eqs. (13)-(15). Next-to-leading order information connecting the bare mass $m_{t}\left(\Lambda_{\mathrm{f}}\right)$, the cut-off $\Lambda_{\mathrm{f}}$ and the 4-fermion coupling strength $G$, can be obtained by consistently minimizing $\Xi_{\text {eff }}$ at each order in $\left(1 / N_{\mathrm{c}}\right)$-expansion.

$$
\begin{gathered}
\left.\frac{\partial \Xi_{\text {eff }}\left(\varepsilon^{2} ; a\right)}{\partial \varepsilon^{2}}\right|_{\varepsilon^{2}=\varepsilon_{\text {gap }}^{2}}=0, \\
\text { where: } \quad \varepsilon_{\text {gap }}^{2}=\frac{m_{t}^{2}\left(\Lambda_{\mathrm{f}}\right)}{\Lambda_{\mathrm{f}}^{2}}=\varepsilon_{0}^{2}+\frac{1}{N_{\mathrm{c}}} \kappa_{1 \mathrm{~g}}+\mathcal{O}\left(\frac{1}{N_{\mathrm{c}}^{2}}\right) .
\end{gathered}
$$

Inserting (19) into (18), taking into account (13) for $\Xi_{\text {eff }}$ and demanding that the coefficients at each power of $\left(1 / N_{\mathrm{c}}\right)$ are zero, we obtain the following relations:

$$
\begin{gathered}
\left.\frac{\partial \Xi^{(0)}}{\partial \varepsilon^{2}}\right|_{\varepsilon^{2}=\varepsilon_{0}^{2}}=0, \\
\left.\kappa_{1 \mathrm{~g}} \frac{\partial^{2} \Xi^{(0)}}{\partial\left(\varepsilon^{2}\right)^{2}}\right|_{\varepsilon^{2}=\varepsilon_{0}^{2}}+\left.\frac{\partial \Xi^{(1)}}{\partial \varepsilon^{2}}\right|_{\varepsilon^{2}=\varepsilon_{0}^{2}}=0 .
\end{gathered}
$$

The ntl-gap equation (20) determines the change of the ratio $\varepsilon_{\text {gap }}^{2}=m_{t}^{2}\left(\Lambda_{\mathrm{f}}\right) / \Lambda_{\mathrm{f}}^{2}$ due to ntl-effects

$$
\delta\left(\varepsilon^{2}\right)_{\text {gap }}^{(\mathrm{ntl})}=\frac{\kappa_{1 \mathrm{~g}}}{N_{\mathrm{c}}}=-\left[\left.\frac{\partial \Xi^{(1)}}{\partial \varepsilon^{2}}\right|_{\varepsilon^{2}=\varepsilon_{0}^{2}}\right] /\left[\left.N_{\mathrm{c}} \frac{\partial^{2} \Xi^{(0)}}{\partial\left(\varepsilon^{2}\right)^{2}}\right|_{\varepsilon^{2}=\varepsilon_{0}^{2}}\right] .
$$

Next, we turn to mass renormalization corrections: $m_{t}\left(\Lambda_{\mathrm{f}}\right) \mapsto\left(m_{t}\right)_{\text {ren. }}$. It is straightforward to check that there are no leading- $N_{\mathrm{c}}$ contributions to these corrections, so that only the 1-PI diagrams shown in Fig. 3 must be taken into account (cf. also [6]).

$$
\delta\left(\varepsilon^{2}\right)_{\text {ren. }}=\frac{\left(m_{t}^{2}\right)_{\text {ren. }}}{\Lambda_{\mathrm{f}}^{2}}-\frac{m_{t}^{2}\left(\Lambda_{\mathrm{f}}\right)}{\Lambda_{\mathrm{f}}^{2}}=\frac{1}{N_{\mathrm{c}}} \kappa_{1 \mathrm{r}}+\mathcal{O}\left(\frac{1}{N_{\mathrm{c}}^{2}}\right) .
$$

At the ntl-level there are three separate contributions, coming from the Higgs, neutral Goldstone and the charged Goldstone, respectively (cf. Fig. 3)

$$
\delta\left(\varepsilon^{2}\right)_{\mathrm{ren} .}^{(\mathrm{ntl})}=\frac{1}{N_{\mathrm{c}}} \kappa_{1 \mathrm{r}}=\frac{1}{N_{\mathrm{c}}}\left(\kappa_{1 \mathrm{r}}^{(H)}+\kappa_{1 \mathrm{r}}^{(G n)}+\kappa_{1 \mathrm{r}}^{(G c h)}\right) .
$$

Calculations and summations of the diagrams of Fig. 3 in Euclidean space yield

$$
\kappa_{1 \mathrm{r}}^{(H)}=-\frac{a}{4} \int_{0}^{\Lambda_{\mathrm{b}}^{2} / \Lambda_{\mathrm{f}}^{2}} \frac{d \bar{p}^{2}}{\left[1-a \mathcal{J}_{H}\left(\bar{p}^{2} ; \varepsilon_{0}^{2}\right)\right]}\left[\left(\sqrt{\bar{p}^{2}\left(\bar{p}^{2}+4 \varepsilon_{0}^{2}\right)}-\bar{p}^{2}\right)\left(2+\frac{\bar{p}^{2}}{2 \varepsilon_{0}^{2}}\right)-\bar{p}^{2}\right],
$$




$$
\begin{aligned}
& \kappa_{1 \mathrm{r}}^{(G n)}=+\frac{a}{4} \int_{0}^{\Lambda_{\mathrm{b}}^{2} / \Lambda_{\mathrm{f}}^{2}} \frac{d \bar{p}^{2}}{\left[1-a \mathcal{J}_{G n}\left(\bar{p}^{2} ; \varepsilon_{0}^{2}\right)\right]} \frac{1}{2 \varepsilon_{0}^{2}} \bar{p}^{2}\left[\bar{p}^{2}+2 \varepsilon_{0}^{2}-\sqrt{\bar{p}^{2}\left(\bar{p}^{2}+4 \varepsilon_{0}^{2}\right)}\right] \\
\kappa_{1 \mathrm{r}}^{(G c h)=}+ & +\frac{a}{4}\left\{\int_{0}^{-\varepsilon_{0}^{2}} \frac{d \bar{p}^{2} \bar{p}^{2}\left[2+\bar{p}^{2} / \varepsilon_{0}^{2}\right]}{\left[1-a \mathcal{J}_{G c h}\left(\bar{p}^{2} ; \varepsilon_{0}^{2}\right)\right]}-\varepsilon_{0}^{2} \int_{-\varepsilon_{0}^{2}}^{\Lambda_{\mathrm{b}}^{2} / \Lambda_{\mathrm{f}}^{2}} d \bar{p}^{2}\left[\frac{1}{\left(1-a \mathcal{J}_{G c h}\left(\bar{p}^{2} ; \varepsilon_{0}^{2}\right)\right)}-\frac{2}{\bar{p}^{2} a \ln \left(1+1 / \varepsilon_{0}^{2}\right)}\right]\right. \\
& \left.-\frac{2 \varepsilon_{0}^{2}}{a \ln \left(1+1 / \varepsilon_{0}^{2}\right)}\left[-\ln \varepsilon_{0}^{2}+\ln \left(\Lambda_{\mathrm{b}}^{2} / \Lambda_{\mathrm{f}}^{2}\right)\right]\right\} .
\end{aligned}
$$

The expressions above were obtained by summing up the corresponding Green functions of Fig. 3, assuming first a (normalized) Euclidean momentum $\bar{q}^{2}>0$ for the external top quark line. Then the analytic continuation to the (approximate) on-shell values $\bar{q}^{2}=-q^{2}=-m_{t}^{(0) 2} / \Lambda_{\mathrm{f}}^{2}\left(=-\varepsilon_{0}^{2}\right)$ had to be performed. In the case of $\kappa_{1 \mathrm{r}}^{(H)}$ and $\kappa_{1 \mathrm{r}}^{(G n)}$, it turned out that this continuation is equivalent to the simple substitution in the Euclidean integrands: $\bar{q}^{2} \mapsto-\varepsilon_{0}^{2}$. The contribution $\kappa_{1 \mathrm{r}}^{(\text {Gch })}$ of the charged Goldstones leading to (26) is somewhat more complicated due to the fact that the massless Goldstone pole at $\bar{p}^{2}=0$ generates a logarithmic branch cut in $\kappa_{1 \mathrm{r}}^{(G c h)}\left(\bar{q}^{2}\right)$ at the threshold value $\bar{q}^{2}=0$. The analytic continuation follows then the usual prescription: $\ln \bar{q}^{2} \mapsto \ln \left(-q^{2}-i \epsilon\right) \mapsto \ln q^{2}-i \pi^{2}$, for $q^{2}>0$. The real part of this term was written in (26) as a separate $\ln \varepsilon_{0}^{2}$-term. Therefore, none of the remaining integrals in $(26)$ is singular.

A few comments are in order here. Eq. (21) shows that the magnitude of the ntl-corrections $\delta\left(\varepsilon^{2}\right)_{\text {gap }}^{(\mathrm{ntl})}$ depends strongly on $\varepsilon_{0}^{2}$, i.e., the solution of the leading- $N_{\mathrm{c}}$ gap equation. This is consistent, since all our calculations were carried out in the " $\left(1 / N_{\mathrm{c}}\right)$-perturbative" manner. The integrals involved in (21) contain no singularities. On the other hand, if we were to relax the large- $N_{\mathrm{c}}$ expansion (19) and solve (18) (with: $\Xi_{\text {eff }}=\Xi^{(0)}+\Xi^{(1)} / N_{\mathrm{c}}$ ) without assuming (19), we would encounter singularities in the integrals over $\bar{p}^{2}$, suggesting that such an approach does not guarantee the masslessness of the Goldstones. These singularities would correspond to the appearance of small nonzero squares of masses for the Goldstones, and they would cancel away only when higher order terms $\mathcal{O}\left(1 / N_{\mathrm{c}}^{2}\right)$ were included in $\Xi_{\text {eff. }}$ Analogous considerations apply also to $\delta\left(\varepsilon^{2}\right)_{\text {ren. }}$.

We have numerically calculated the ntl-changes (21) and (23), based on the integrals (14)-(15) and (24)-(26). The integrals over the squares $\bar{p}^{2}$ of the normalized bosonic momenta were performed using the following explicit expressions for the normalized 2-point Green functions (16)

$$
\begin{aligned}
\mathcal{J}_{\text {Gch }}\left(\bar{p}^{2} ; \varepsilon^{2}\right) & =\left[1-\frac{3}{4} \bar{p}^{2}-\frac{1}{2} \varepsilon^{2}+\frac{1}{2 \bar{p}^{2}}\left(\bar{p}^{2}+\varepsilon^{2}\right)^{2} \ln \left(\bar{p}^{2}+\varepsilon^{2}\right)-\frac{1}{2}\left(2 \varepsilon^{2}+\bar{p}^{2}\right) \ln \left(1+\varepsilon^{2}\right)-\frac{\varepsilon^{4}}{2 \bar{p}^{2}} \ln \varepsilon^{2}\right] \\
\mathcal{J}_{H}\left(\bar{p}^{2} ; \varepsilon^{2}\right) & =\mathcal{D}\left(\bar{p}^{2} ; \varepsilon^{2}\right)-\frac{\left(\bar{p}^{2}+4 \varepsilon^{2}\right)}{4} \mathcal{C}\left(\bar{p}^{2} ; \varepsilon^{2}\right) \\
\mathcal{J}_{G n}\left(\bar{p}^{2} ; \varepsilon^{2}\right) & =\mathcal{D}\left(\bar{p}^{2} ; \varepsilon^{2}\right)-\frac{\bar{p}^{2}}{4} \mathcal{C}\left(\bar{p}^{2} ; \varepsilon^{2}\right)
\end{aligned}
$$


where we have defined:

$$
\begin{aligned}
& \mathcal{D}\left(\bar{p}^{2} ; \varepsilon^{2}\right)=\left[\frac{3}{4}-\frac{\varepsilon^{2}}{2} \ln \left(\varepsilon^{-2}+1\right)+\frac{1}{8 \bar{p}^{2}}\left(1+\varepsilon^{2}\right)^{2}-\frac{\bar{p}^{2}}{8}-\frac{1}{8 \bar{p}^{2}}\left(1-\bar{p}^{2}+\varepsilon^{2}\right) \mathcal{B}-\frac{\varepsilon^{2}}{2} \ln \left(\frac{a_{3}}{2 \varepsilon^{2}}\right)\right] \\
& \mathcal{C}\left(\bar{p}^{2} ; \varepsilon^{2}\right)=\left[1+\frac{1}{\bar{p}^{2}}\left(1+\varepsilon^{2}-\mathcal{B}\right)+(1-\mathcal{A}) \ln \left(\varepsilon^{-2}+1\right)+\mathcal{A} \ln \left(\frac{a_{1}}{a_{2}}\right)+\ln \left(\frac{a_{3}}{2 \varepsilon^{2}}\right)\right] .
\end{aligned}
$$

The parameters $\mathcal{A}, \mathcal{B}$ and $a_{j}(j=1,2,3)$ denote the expressions

$$
\begin{gathered}
\mathcal{A}=\sqrt{1+4 \frac{\varepsilon^{2}}{\bar{p}^{2}}}, \quad \mathcal{B}=\sqrt{\left(1-\bar{p}^{2}+\varepsilon^{2}\right)^{2}+4 \bar{p}^{2} \varepsilon^{2}}, \\
a_{1}=\left(\bar{p}^{2}+3 \varepsilon^{2}-1+\mathcal{A B}\right), \quad a_{2}=\bar{p}^{2}+3 \varepsilon^{2}+\left(\bar{p}^{2}+\varepsilon^{2}\right) \mathcal{A}, \quad a_{3}=1-\bar{p}^{2}+\varepsilon^{2}+\mathcal{B} .
\end{gathered}
$$

The partial derivatives $\partial \mathcal{J}_{X} / \partial \varepsilon^{2}(X=H, G n, G c h)$, needed for the calculation of the integrand of $\partial \Xi^{(1)} / \partial \varepsilon^{2}$ in the ntl-gap equation $(20)-(21)$, are obtained directly from (27).

The input value for the integrations was the parameter $a=N_{\mathrm{c}} G \Lambda_{\mathrm{f}}^{2} / 8 \pi^{2}$ of (13) and (17), which is essentially a dimensionless measure of the strength of the original 4-fermion coupling $G$ in (11). We also had to choose a specific value of the ratio of the cut-offs $\Lambda_{\mathrm{b}} / \Lambda_{\mathrm{f}}(=\mathcal{O}(1))$. As suggested by the diagrams of Fig. 2, realistic choices in the present framework of simple spherical cut-offs have: $\bar{p}_{\max }^{2} \leq \bar{k}_{\max }^{2}$, which implies $\Lambda_{\mathrm{b}} / \Lambda_{\mathrm{f}} \lesssim 1$. We have made two choices: $\Lambda_{\mathrm{b}} / \Lambda_{\mathrm{f}}=1 / \sqrt{2}(\approx 0.707), 0.5$. It turned out that the ntl-effects (21) and (23) decrease the ratio $\left(m_{t}^{\text {phys. }} / \Lambda_{\mathrm{f}}\right)^{2}$ when compared to the leading- $N_{\mathrm{c}}$ expression $\varepsilon_{0}^{2}$ of (17) (note: at the leading- $N_{\mathrm{c}}$ level we have $m_{t}^{(0)}=m_{t}^{\text {phys. }}$, and at the ntl-level we have $\left.m_{t}^{\text {ren. }}=m_{t}^{\text {phys. }}\right)$. Stability of the results requires that the ntl-changes of the ratio $m_{t}^{\text {phys. }} / \Lambda_{\mathrm{f}}$ not be too large, so that the $\left(1 / N_{\mathrm{c}}\right)$-expansion would have some qualitative predictive power. This implies that the parameter $a$, or equivalently the leading- $N_{\mathrm{c}}$ ratio $\varepsilon_{0}=m_{t}^{(0)} / \Lambda_{\mathrm{f}}$ (cf. (17)), cannot decrease beyond a certain critical value, and that the resulting ratio $m_{t}^{\text {ren. }} / \Lambda_{\mathrm{f}}$ cannot be smaller than a critical value $\left(m_{t}^{\text {ren. }} / \Lambda_{\mathrm{f}}\right)_{\text {crit. }}$ correspondingly. Consequently, the cut-off $\Lambda_{\mathrm{f}}$ cannot exceed an upper bound $\left(\Lambda_{\mathrm{f}}\right)_{\max }$ (we took: $m_{t}^{\text {ren. }}=m_{t}^{\text {phys. }}=180 \mathrm{GeV}$ ). Specifically, we demanded that the value of $m_{t}^{\text {phys. }} / \Lambda_{\mathrm{f}}$ be diminished by the ntl-effects (21) and (23) not more than by a factor of: $\sqrt{2}, 2,3,4$. The resulting critical values of ratios and of cut-offs are given in Table 1 (columns 3-6), where, in addition, we included in the last four columns the results when only the Higgs effects (without Goldstones) were taken into account. Comparing the two sets of results, we conclude that the Goldstone degrees of freedom change the numbers substantially. However, in both cases, we are led to the same qualitative conclusion: the cut-off $\Lambda_{\mathrm{f}}$ does not surpass $\mathcal{O}(1 \mathrm{TeV})$. For $\Lambda_{\mathrm{b}} / \Lambda_{\mathrm{f}}=1$ the calculations show that the negative ntl-contribution $\delta\left(\varepsilon^{2}\right)^{(\mathrm{ntl})}\left(=\delta\left(\varepsilon^{2}\right)_{\text {gap }}^{(\mathrm{ntl})}+\delta\left(\varepsilon^{2}\right)_{\mathrm{ren} .}^{(\mathrm{ntl})}\right)$ is under the inclusion of the Goldstone contributions always stronger than the leading- $N_{\mathrm{c}}$ one: $\left|\delta\left(\varepsilon^{2}\right)^{(\mathrm{ntl})}\right|>\varepsilon_{0}^{2}$. 
Looking more closely upon the contributions of the various degrees of freedom to the "gap" ntl-

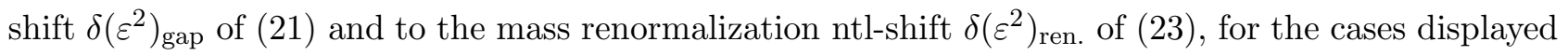
in Table 1, the following picture emerges: the Higgs and each one of the three Goldstone degrees of freedom contribute comparable negative values to $\delta\left(\varepsilon^{2}\right)_{\text {gap }}$; the Higgs and the charged Goldstone degrees of freedom contribute each a negative value and the neutral Goldstone a weaker positive value to $\delta\left(\varepsilon^{2}\right)_{\text {ren. }}$, leading thus to a negative $\delta\left(\varepsilon^{2}\right)_{\text {ren. }}$. Consequently, both $\delta\left(\varepsilon^{2}\right)_{\text {gap }}$ and $\delta\left(\varepsilon^{2}\right)_{\text {ren. }}$ are negative, and $\left|\delta\left(\varepsilon^{2}\right)_{\text {gap }}\right|$ is larger than $\left|\delta\left(\varepsilon^{2}\right)_{\text {ren. }}\right|$, usually by more than a factor of $2\left(\delta\left(\varepsilon^{2}\right)_{\text {gap }} \approx-0.3,-0.15\right.$, -0.05 , for $\Lambda_{\mathrm{b}} / \Lambda_{\mathrm{f}}=1,0.707,0.5$, respectively). It turns out that $\left|\delta\left(\varepsilon^{2}\right)_{\text {ren. }}\right| / \varepsilon_{0}^{2} \lesssim 0.3$ when $\varepsilon_{0}^{2} \rightarrow 0$. On the other hand, $\delta\left(\varepsilon^{2}\right)_{\text {gap }}$ remains relatively stable as $\varepsilon_{0}^{2} \rightarrow 0 ; \delta\left(\varepsilon^{2}\right)_{\text {gap }}$ is thus identified as the source of the observed " $1 / N_{\mathrm{c}}$-nonperturbative" behavior, unlike $\delta\left(\varepsilon^{2}\right)_{\text {ren. }}$.

Finally, the leading part of QCD effects was included. The "gap" part is represented by the contributions coming from the diagrams of Fig. 2, where the internal dashed lines represent now the gluon propagators (in Landau gauge). The momentum integrals were regulated by means of a propertime cut-off $1 / \Lambda_{\mathrm{f}}^{2}$ for the quarks and $1 / \Lambda_{\mathrm{b}}^{2}$ for the gluons. The corresponding contribution to $\Xi^{(1)}$ to be added in (15) was derived in [6]

$$
\Xi^{(1 ; \mathrm{gl})}\left(\varepsilon^{2} ; \Lambda_{\mathrm{b}}^{2} / \Lambda_{\mathrm{f}}^{2} ; a_{\mathrm{gl}}\right)=2 \int_{0}^{\Lambda_{\mathrm{b}}^{2} / \Lambda_{\mathrm{f}}^{2}} d \bar{p}^{2} \bar{p}^{2} \ln \left[1-a_{\mathrm{gl}} \mathcal{J}_{\mathrm{gl}}\left(\bar{p}^{2} ; \varepsilon^{2}\right)\right],
$$

Above, we denoted by $a_{\mathrm{gl}}$ the QCD coupling parameter: $a_{\mathrm{gl}}=3 \alpha_{s}\left(m_{t}\right) / \pi \approx 0.105$. The (proper-time regulated) 2-point Green function $\mathcal{J}_{\text {gl }}$ appearing in (30) is

$$
\begin{aligned}
\mathcal{J}_{\mathrm{gl}}\left(\bar{p}^{2}, \varepsilon^{2}\right)= & -\frac{1}{6}\left(2 \frac{\varepsilon^{2}}{\bar{p}^{2}}-1\right) \mathcal{E}\left(\frac{\varepsilon^{2}}{\bar{p}^{2}}\right)+\frac{1}{6} \ln \varepsilon^{2}+\frac{2}{9} \\
& -\frac{1}{6}\left(\frac{\bar{p}^{2}}{5}+\varepsilon^{2}\right)+\frac{1}{4}\left(\frac{\bar{p}^{4}}{140}+\frac{\bar{p}^{2} \varepsilon^{2}}{15}+\frac{\varepsilon^{4}}{6}\right)+\mathcal{O}\left(\bar{p}^{6}, \varepsilon^{6}\right),
\end{aligned}
$$

where we denoted by $\mathcal{E}$ the integral

$$
\mathcal{E}(w)=\int_{0}^{1} d z \ln \left[1+\frac{z(1-z)}{w}\right]=-2+\sqrt{(4 w+1)} \ln \left[\frac{\sqrt{(4 w+1)}+1}{\sqrt{(4 w+1)}-1}\right] .
$$

We point out that expression (30), unlike (15), turns out to be numerically almost equal to its 2-loop approximation (obtained by the replacement: $\ln \left[1-a_{\mathrm{gl}} \mathcal{J}_{\mathrm{gl}}\left(\bar{p}^{2}, \varepsilon^{2}\right)\right] \mapsto-a_{\mathrm{gl}} \mathcal{J}_{\mathrm{gl}}\left(\bar{p}^{2}, \varepsilon^{2}\right)$ ), the difference being only a fraction of a percent.

The leading QCD $m_{t}$-mass renormalization effect comes from the 2-loop version of the diagrams of Fig. 3, where the dashed line is now the gluonic propagator. The proper-time cut-off gives (cf. [6])

$$
\delta\left(\varepsilon^{2}\right)_{\text {ren. }}^{\mathrm{QCD}}=\frac{2}{3} a_{\mathrm{gl}} \varepsilon_{0}^{2}\left[\ln \left(\varepsilon_{0}^{-2}\right)+\ln \left(\Lambda_{\mathrm{b}}^{2} / \Lambda_{\mathrm{f}}^{2}\right)+0.256 \ldots+\frac{5 \Lambda_{\mathrm{f}}^{2}}{9 \Lambda_{\mathrm{b}}^{2}} \varepsilon_{0}^{2}+\mathcal{O}\left(\varepsilon_{0}^{4}\right)\right] .
$$


This expression is to be added to (23) in order to obtain the QCD-modified $\delta\left(\varepsilon^{2}\right)_{\text {ren. }}$.

QCD effects give positive contributions to $\delta\left(\varepsilon^{2}\right)_{\text {gap }}$ and to $\delta\left(\varepsilon^{2}\right)_{\text {ren. }}$. The contribution to the "gap" ntl-shift $\delta\left(\varepsilon^{2}\right)_{\text {gap }}$ is by about one order of magnitude smaller than the corresponding contribution of the scalars to this quantity. On the other hand, the positive QCD contribution to $\delta\left(\varepsilon^{2}\right)_{\text {ren. }}$ is larger by a factor of $3-5$, and it is comparable to the positive contribution of the neutral Goldstone to this quantity. Altogether, $\delta\left(\varepsilon^{2}\right)_{\text {ren. }}$ is still clearly negative under the inclusion of QCD effects, and $\delta\left(\varepsilon^{2}\right)_{\text {gap }}$ remains negative and with a substantially larger magnitude than $\delta\left(\varepsilon^{2}\right)_{\text {ren. }}$ (by a factor of 2 or more). In Table 2, we display the results analogous to those of Table 1, but now these QCD effects are included. Comparing Table 1 and Table 2, we see that the inclusion of QCD changes the results rather modestly. The basic result remains the same: as long as we demand that the leading- $N_{\mathrm{c}}$ gap equation have at least a qualitatively predictive power, the cut-off $\Lambda_{\mathrm{f}}\left(\sim \Lambda_{\mathrm{b}}\right)$ cannot surpass $\mathcal{O}(1 \mathrm{TeV})$.

In conclusion, we mention that other authors have studied ntl-effects in the TSM and in related frameworks [9]- [11]. The authors of [9] calculated ntl-contributions to critical exponents of the fields within NJL-type models at the fixed point, i.e., at the location of the non-trivial zero of $\beta$-function, for various dimensions $d$. The implications of [9] in relation to 4-dimensional NJL-type models at low energy and with finite cut-off are not clear and would deserve investigation. When concluding the present work, a somewhat related work by K. Akama [10] came to our attention. Akama investigates the ntl-effects by considering the compositeness condition, i.e., the condition that the renormalization constants of a composite scalar field and of its self-interaction parameter are zero. He reaches the conclusion that the ntl-effects for $N_{\mathrm{c}}=3$ are substantially stronger than the leading- $N_{\mathrm{c}}$ contributions and lead to physically unacceptable results: negative Higgs mass, negative $\Phi^{4}$-coupling, etc. Furthermore, Lurié and Tupper [1] had earlier considered the compositeness condition and took into account at least some of the effects beyond the leading- $N_{\mathrm{c}}$, arriving at qualitatively the same conclusion as Akama - that $1 / N_{\mathrm{c}}$-expansion diverges. We note that these three authors treated the TSM as a renormalizable Yukawa-type model (without gauge bosons) plus the compositeness condition, similar to (but not identical with) the approach of BHL [3]. Thus, they implicitly assumed large cut-offs $\Lambda$, in the sense that $\ln \Lambda$-terms would entirely dominate over the $\Lambda$-independent parts. Consequently, the results of Akama, Lurié and Tupper appear to not contradict the results of the present paper - i.e., that the TSM can be interpreted at the ntl-level in a straightforward manner only if $\Lambda=\mathcal{O}(1 \mathrm{TeV})$, and that it may be difficult or impossible to interpret the model if $\Lambda>\mathcal{O}(1 \mathrm{TeV})$. 


\section{Acknowledgment}

This work was supported in part by the Deutsche Forschungsgemeinschaft and in part by the European Union Project CHRX-CT92-0026.

\section{References}

[1] Y. Nambu, Proceedings of the Kazimierz Conference "New Theories in Physics" (1988), pp. 1-10; V.A. Miransky, M. Tanabashi and K. Yamawaki, Mod. Phys. Lett. A 4 (1989) 1043; Phys. Lett. B 221 (1989) 177.

[2] W.J. Marciano, Phys. Rev. Lett. 62 (1989) 2793; Phys. Rev. D 41 (1990) 219.

[3] W. A. Bardeen, C. T. Hill and M. Lindner, Phys. Rev. D 41 (1990) 1647.

[4] M. Bando, T. Kugo, N. Maekawa, N. Sasakura and Y. Watabiki, Phys. Lett. B 246 (1990) 466; A. Hasenfratz, P. Hasenfratz, K. Jansen, J. Kuti and Y. Shen, Nucl. Phys. B 365 (1991) 79; S.F. King and S.H. Mannan, Phys. Lett. B 241 (1990) 249; Z. Phys. C 52 (1991) 59; C.T. Hill, M.A. Luty and E.A. Paschos, Phys. Rev. D 43 (1991) 3011; Y. Achiman and A. Davidson, Phys. Lett. B 261 (1991) 431; F. Cooper and J. Pérez-Mercader, Phys. Rev. D 43 (1991) 4129; J. Zinn-Justin, Nucl. Phys. B 367 (1991) 105; P. Fishbane, R.E. Norton and T.N. Truong, Phys. Rev. D 46 (1992) 1768; P. Fishbane and R.E. Norton, Phys. Rev. D 48 (1993) 4924; A. Blumhofer, R. Dawid and M. Lindner, Phys. Lett. B 360 (1995) 123.

[5] Y. Nambu and G. Jona-Lasinio, Phys. Rev. 122 (1961) 345.

[6] G. Cvetič, E.A. Paschos and N.D. Vlachos, Phys. Rev. D 53 (1996) 2820.

[7] T. Kugo, Prog. Theor. Phys. 55 (1976) 2032; K. Kikkawa, ibid. 56 (1976) 947.

[8] S. Weinberg, Phys. Rev. D 7 (1973) 2887.

[9] S. Hands, A. Kocić and J.B. Kogut, Phys. Lett. B 273 (1991) 111; Ann. Phys. 224 (1993) 29; J.A. Gracey, Phys. Lett. B 308 (1993) 65; Z. Phys. C 61 (1994) 115; S.E. Derkachov, N.A. Kivel, A.S. Stepanenko and A.N. Vasil'ev, in "Hadrons-93", Conference proceedings, (publisher: Novy Svit, 1993); Saclay preprint SACLAY-SPHT-93-016 (hep-th/9302034).

[10] K. Akama, Phys. Rev. Lett. 76 (1996) 184.

[11] D. Lurié and G.B. Tupper, Phys. Rev. D 47 (1993) 3580. 
Table 1

\begin{tabular}{|l|l||c|c|c|c||c|c|c|c|}
\hline$m_{t}^{\text {ren. }} / m_{t}^{(0)}$ & $\Lambda_{\mathrm{b}} / \Lambda_{\mathrm{f}}$ & $\begin{array}{c}m_{t}^{(0)} / \Lambda_{\mathrm{f}} \\
(\mathrm{sc})\end{array}$ & $\begin{array}{c}m_{t}^{\text {ren. }} / \Lambda_{\mathrm{f}} \\
(\mathrm{sc})\end{array}$ & $\begin{array}{c}\Lambda_{\mathrm{f}}(\mathrm{sc}) \\
{[\mathrm{TeV}]}\end{array}$ & $\begin{array}{c}\Lambda_{\mathrm{b}}(\mathrm{sc}) \\
{[\mathrm{TeV}]}\end{array}$ & $\begin{array}{c}m_{t}^{(0)} / \Lambda_{\mathrm{f}} \\
(H)\end{array}$ & $\begin{array}{c}m_{t}^{\text {ren. }} / \Lambda_{\mathrm{f}} \\
(H)\end{array}$ & $\begin{array}{c}\Lambda_{\mathrm{f}}(H) \\
{[\mathrm{TeV}]}\end{array}$ & $\begin{array}{c}\Lambda_{\mathrm{b}}(H) \\
{[\mathrm{TeV}]}\end{array}$ \\
\hline \hline $1 / \sqrt{2}(=0.707)$ & $1 / \sqrt{2}$ & - & - & - & - & 0.320 & 0.226 & 0.79 & 0.56 \\
0.5 & $1 / \sqrt{2}$ & 0.757 & 0.379 & 0.48 & 0.34 & 0.250 & 0.125 & 1.44 & 1.02 \\
0.333 & $1 / \sqrt{2}$ & 0.540 & 0.180 & 1.00 & 0.71 & 0.226 & 0.075 & 2.39 & 1.69 \\
0.25 & $1 / \sqrt{2}$ & 0.500 & 0.125 & 1.44 & 1.02 & 0.219 & 0.055 & 3.28 & 2.32 \\
\hline 0.707 & 0.5 & 0.516 & 0.365 & 0.49 & 0.25 & 0.200 & 0.141 & 1.27 & 0.64 \\
0.5 & 0.5 & 0.329 & 0.164 & 1.09 & 0.55 & 0.161 & 0.081 & 2.24 & 1.12 \\
0.333 & 0.5 & 0.282 & 0.094 & 1.91 & 0.96 & 0.147 & 0.049 & 3.67 & 1.84 \\
0.25 & 0.5 & 0.270 & 0.067 & 2.67 & 1.34 & 0.143 & 0.036 & 5.04 & 2.52 \\
\hline
\end{tabular}


Table 2

\begin{tabular}{|l|l||c|c|c|c|}
\hline$m_{t}^{\text {ren. }} / m_{t}^{(0)}$ & $\Lambda_{\mathrm{b}} / \Lambda_{\mathrm{f}}$ & $m_{t}^{(0)} / \Lambda_{\mathrm{f}}$ & $\begin{array}{c}m_{t}^{\text {ren. }} / \Lambda_{\mathrm{f}} \\
(\mathrm{sc}+\mathrm{gl})\end{array}$ & $\begin{array}{c}\Lambda_{\mathrm{f}}(\mathrm{sc}+\mathrm{gl}) \\
{[\mathrm{TeV}]}\end{array}$ & $\begin{array}{c}\Lambda_{\mathrm{b}}(\mathrm{sc}+\mathrm{gl}) \\
{[\mathrm{TeV}]}\end{array}$ \\
\hline \hline $1 / \sqrt{2}(=0.707)$ & $1 / \sqrt{2}$ & - & - & - & - \\
0.5 & $1 / \sqrt{2}$ & 0.641 & 0.321 & 0.56 & 0.40 \\
0.333 & $1 / \sqrt{2}$ & 0.461 & 0.154 & 1.17 & 0.83 \\
0.25 & $1 / \sqrt{2}$ & 0.428 & 0.107 & 1.68 & 1.19 \\
\hline 0.707 & 0.5 & 0.466 & 0.329 & 0.55 & 0.27 \\
0.5 & 0.5 & 0.288 & 0.144 & 1.25 & 0.63 \\
0.333 & 0.5 & 0.248 & 0.083 & 2.18 & 1.09 \\
0.25 & 0.5 & 0.237 & 0.059 & 3.03 & 1.52 \\
\hline
\end{tabular}

\section{Table and figure captions}

Table 1: The quark (fermion) cut-offs $\Lambda_{\mathrm{f}}$ and the bosonic cut-offs $\Lambda_{\mathrm{b}}$ which result when we impose the requirement that the ratio of the next-to-leading $m_{t}$ (i.e., $m_{t}^{\text {ren. }) ~ t o ~ t h e ~ l e a d i n g-~} N_{\mathrm{c}} m_{t}^{(0)}$ be $1 / \sqrt{2}, 1 / 2,1 / 3$ and $1 / 4$, respectively; we have chosen the cut-off ratios $\Lambda_{\mathrm{b}} / \Lambda_{\mathrm{f}}=1 / \sqrt{2}, 1 / 2$; "sc" indicates quantities for the case when all four scalar degrees were taken into account at the next-to-leading (ntl) level; " $H$ " indicates quantities when only the physical Higgs degree of freedom was taken into account at the ntl level. We identify $m_{t}^{\text {ren. }}=m_{t}^{\text {phys. }}=180 \mathrm{GeV}$. No entries in the first line for the "sc" case mean that $\left(m_{t}^{\text {ren. }} / m_{t}^{(0)}\right)<0.707$ for any choice of $m_{t}^{(0)} / \Lambda_{\mathrm{f}}\left(\right.$ when $\left.\Lambda_{\mathrm{b}} / \Lambda_{\mathrm{f}}=0.707\right)$.

Table 2: Same as Table 2, but this time for the case when, in addition, the leading part of the QCD (two loop) effects was taken into account.

Figs. 1(a)-(c): The 1-loop 1-PI diagrams contributing to 1-PI Green functions $\tilde{\Gamma}_{H}^{(2 m ; 1)}\left(p_{1}, \ldots, p_{2 m}\right)$, which in turn yield the leading- $N_{\mathrm{C}}$ part $V_{\mathrm{eff}}^{(0)}$ in the formal $1 / N_{\mathrm{C}}$-expansion of $V_{\text {eff }}$. Full lines represent massless top quarks, and dotted external lines the scalar non-dynamical Higgs of the Lagrangian (3).

Fig. 2: The $(\ell+1)$-loop 1-PI diagrams which contribute to the 1-PI Green functions which in turn yield the ntl-part $V_{\mathrm{eff}}^{(1)}$ (beyond 1-loop) in the formal $1 / N_{\mathrm{c}}$-expansion of $V_{\text {eff. }}$. The diagrams contain $\ell$ loops of (massless) quarks. These loops are connected into another circle by $\ell$ propagators of the (non-dynamical) scalars (all either Higgs, or neutral Goldstone, or charged Goldstone). In the case of charged Goldstone propagators, the quark loops are made up of the top and the 
bottom quark.

Figs. 3(a)-(c): the 1-PI diagrams with two external top quark legs which give the leading $\left(\mathcal{O}\left(1 / N_{\mathrm{C}}\right)\right)$ contribution to the renormalization of the mass $m_{t}$. Unlike the diagrams of Figs. 1-2, the top quark propagators here contain the non-zero bare mass $m_{t}$ which was the solution to the leading- $N_{\mathrm{C}}$ gap equation. The dashed lines are all the non-dynamical scalars (either the Higgs, or the neutral Goldstones, or the charged Goldstones). For the case of charged Goldstone propagators, the loops contain one massive top quark and one massless bottom quark. 


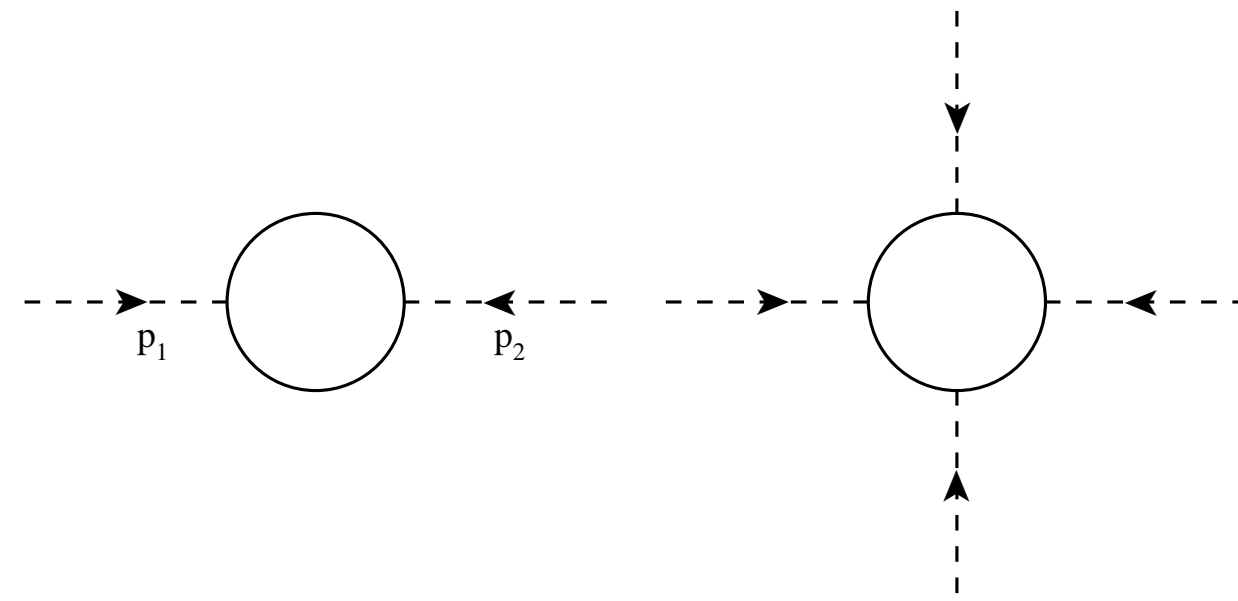

Fig. 1a
Fig. 1b

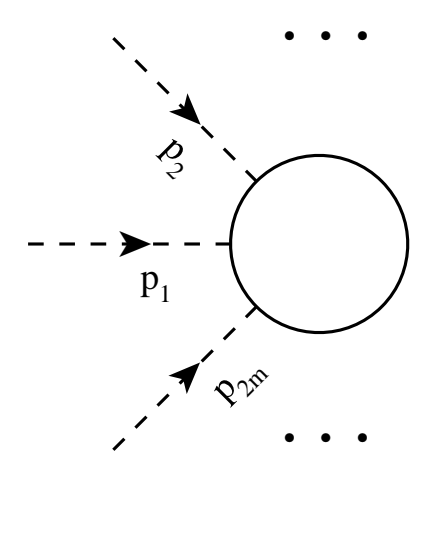

Fig. 1c 


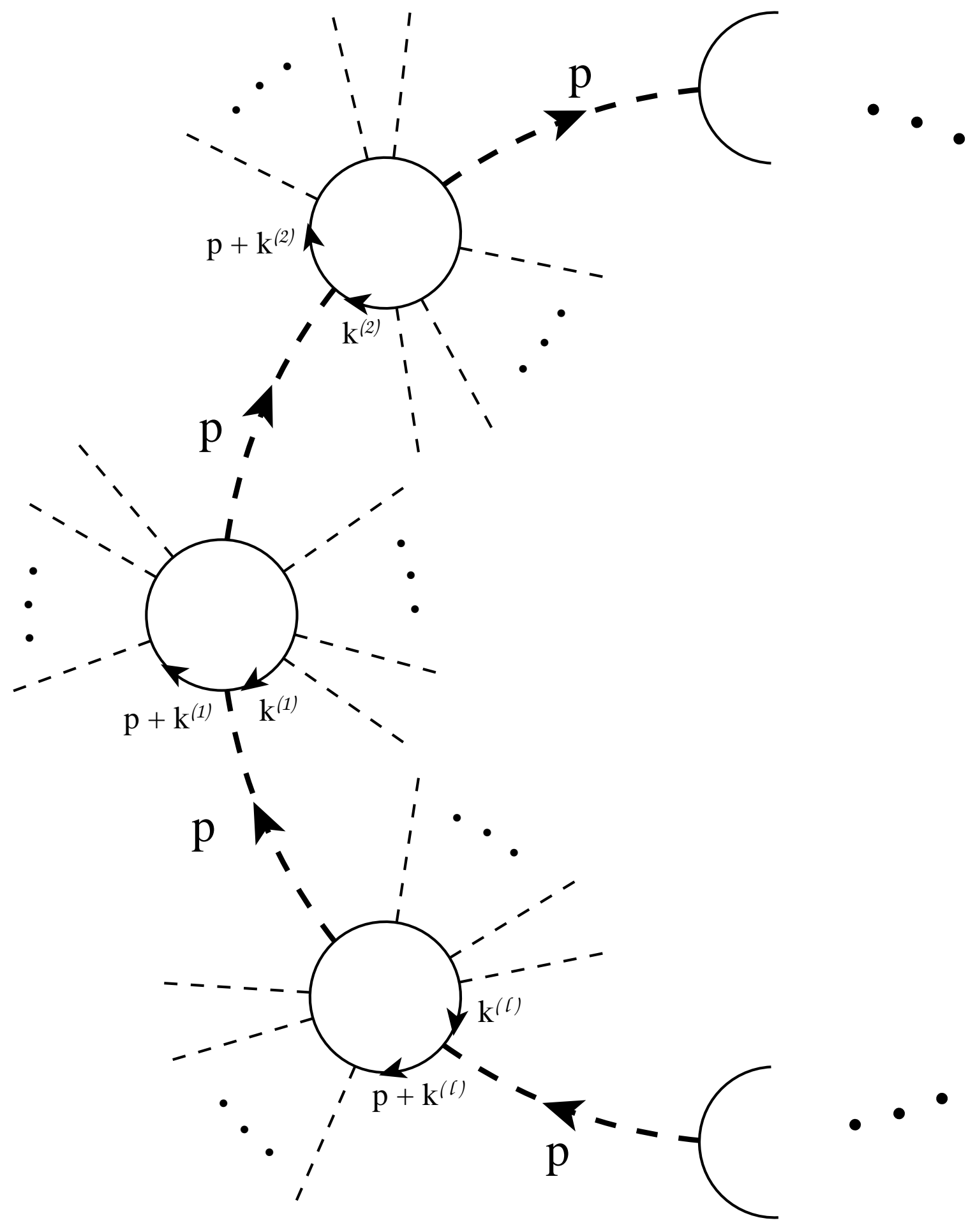

Fig. 2 


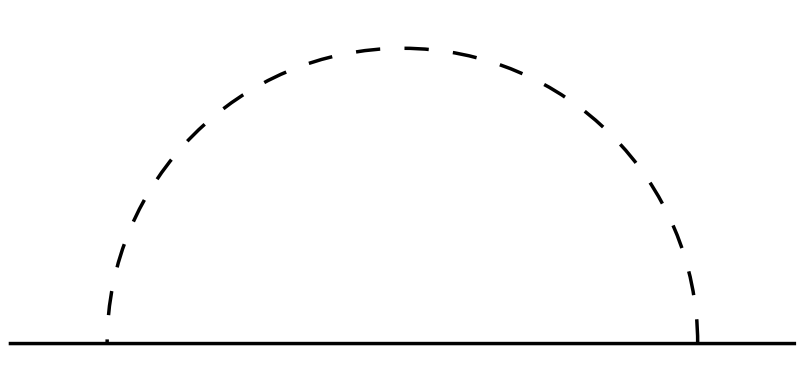

Fig. 3a

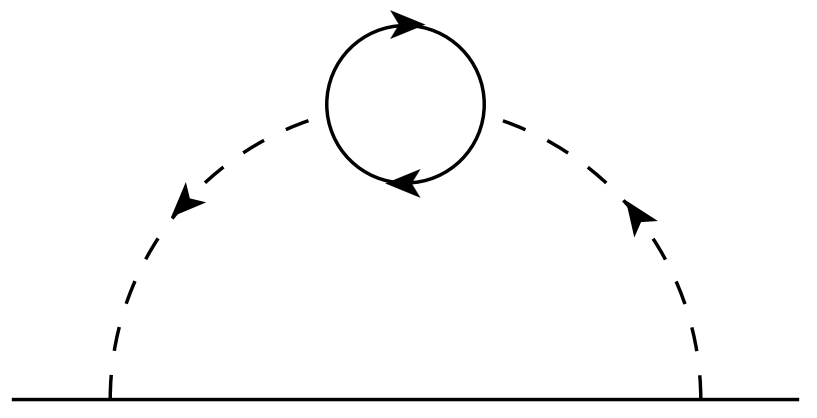

Fig. 3b

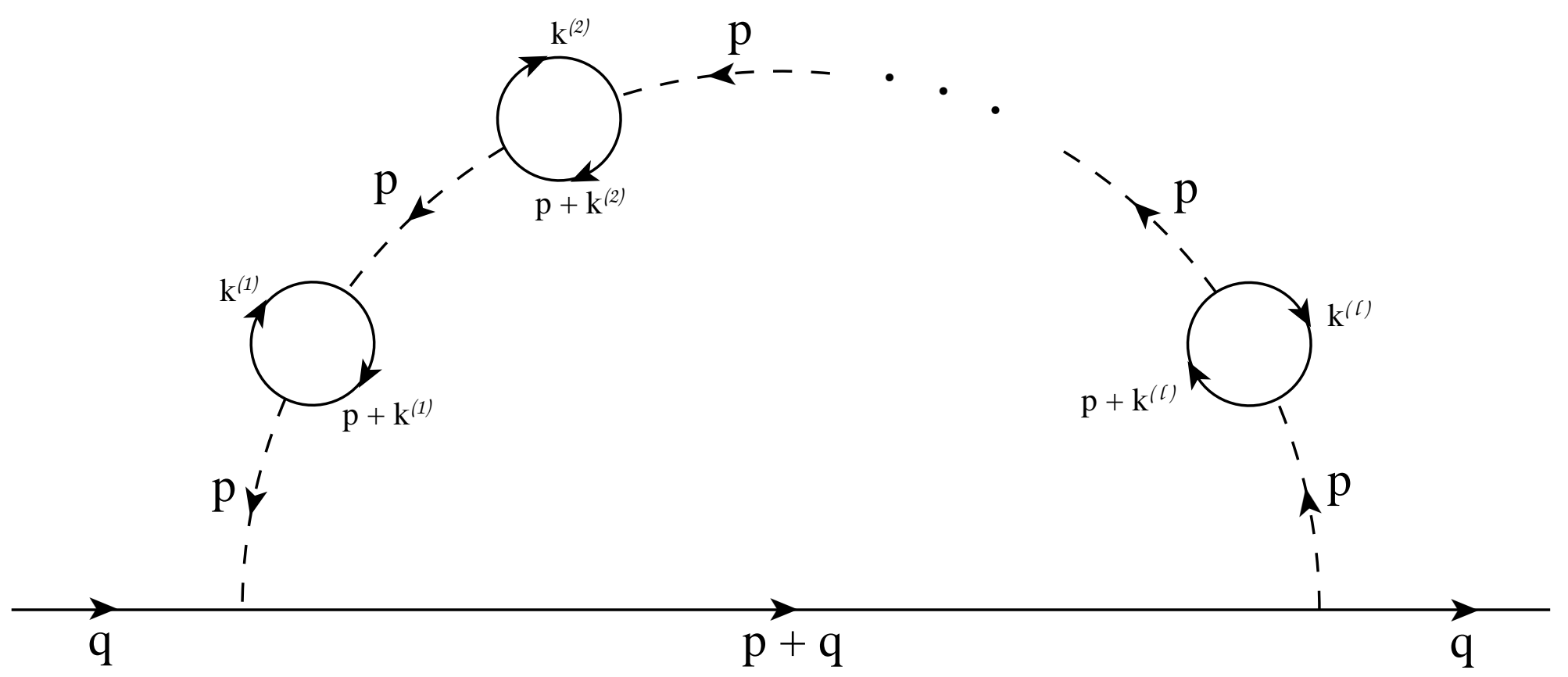

Fig. 3c 